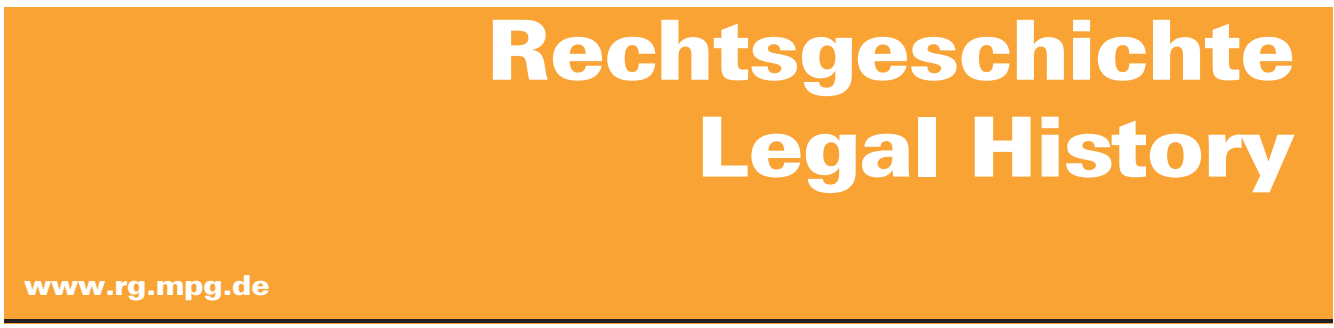

http://www.rg-rechtsgeschichte.de/rg24

$\operatorname{Rg} 242016$

$402-411$

Zitiervorschlag: Rechtsgeschichte - Legal History Rg 24 (2016)

http://dx.doi.org/10.12946/rg24/402-411

\title{
Gerhard Melinz
}

\section{Sozialrechtsgeschichte in Österreich: Status quo und quo vadis?}


Gerhard Melinz

\section{Sozialrechtsgeschichte in Österreich: Status quo und quo vadis?*}

1 Wissenschaftsdisziplinen und Sozialrechtsgeschichtsschreibung

Ein Überblick über die Sozialrechtsgeschichtsschreibung und ihre möglichen Innovationen in Österreich stellt eine besondere Herausforderung dar. Eigentlich existiert hierzulande keine universitäre Sozialrechtshistorikerzunft. Die Kombination von Rechtsgeschichte und Sozialrecht scheint ganz offenkundig wenig einladend zu sein, aus welchen Gründen auch immer. Die Rechtshistoriker ${ }^{1}$ beschäftig(t)en sich mit unterschiedlichen Themen und Untersuchungszeiträumen, wobei die Rechtsmaterien des Sozialrechts und das 20. Jahrhundert ganz und gar nicht im Vordergrund der Forschung stehen bzw. standen. ${ }^{2}$ In den Reihen der Arbeits- und Sozialrechtler dominiert das Arbeitsrecht und der Gegenwartsbezug. ${ }^{3}$ Eine simple Historisierung der aktuellen Sozialrechtsforschung ist auch kein wirklich gangbarer Weg. ${ }^{4}$

Der Blick in die jeweiligen Einleitungskapitel von Standardwerken zum Sozialrecht bietet eine gute Möglichkeit, die Berücksichtigung einschlägiger Deutungen des historischen Gewordenseins diverser Sozialrechtsbereiche bzw. des Sozialrechts zur Kenntnis zu nehmen. In Anbetracht dessen, dass den betreffenden Aussagen meist nur bibliographische Blockangaben vorangestellt sind, ist nicht immer klar, auf welcher Quellenbasis die eine oder andere Behauptung beruht oder welcher Publikation sie entnommen ist. Eines ist aber unbestreitbar: Die auf die Gegenwart ausgerichteten Sozialrechtler kümmern sich keineswegs um den sozialrechtsgeschichtlichen Forschungsstand. ${ }^{5}$ Und dies führt auch schon zu der Frage hin, welche themenbezogene Fachliteratur von NichtRechtshistorikern überhaupt erwähnenswert wäre und ist. Unter Rechtshistorikern - zumindest bundesdeutschen - würden quellenbezogen arbeitende Historiker als berücksichtigungswürdig gelten. Gelegentlich hat man den Eindruck, dass Historiker und manchmal auch Sozialwissenschaftler gerade auf dem Gebiet des Sozialrechts einen guten Ruf genießen, weil sie sich mit gewichtigen Publikationen zur historischen Sozialpolitik- oder Wohlfahrtsstaatsforschung präsentieren können. Ganz grundsätzlich sollten immer die Regeln für "gute Forschung « gelten. Angesichts beträchtlicher Forschungsdesiderate im Bereich der Sozialrechtsgeschichte wären hier nicht Kriterien wie Quellberuflichkeit und die Zitierhäufigkeit zu berück-
* Dieser Beitrag entstand im Rahmen eines Forschungsprojektes der Fachhochschule FH Campus Wien.

1 Im Interesse einer besseren Lesbarkeit wird nicht ausdrücklich in geschlechtsspezifischen Personenbezeichnungen differenziert. Die gewählte männliche Form schließt eine adäquate weibliche Form gleichberechtigt ein.

2 Für die Verfassungsrechts- und Verwaltungsrechtsgeschichte finden sich allerdings schon Beiträge. Im Übrigen mögen die mehrfachen politischen Regimewechsel in der ersten Hälfte des 20. Jahrhunderts je nach (partei-)politischer Orientierung des Universitätspersonals auch zur $\mathrm{Zu}$ rückhaltung beigetragen haben; siehe jedoch jetzt ReIter-Zatloukal / ROTHLÄNDER / SCHÖLNBERGER (2012) zum Zeitabschnitt 1933 bis 1938 - veranstaltet von progressiven Rechtshistorikerinnen. Dieser Sammelband verzichtet allerdings auf die Nutzung von Forschungsergebnissen, die in Arbeiten zur Interaktion von erstem und zweitem sozialen Netz gewonnen worden waren, und spiegelt damit den aktuellen Forschungsstand nur unvollkommen wider.

3 Dies verdeutlicht auch ein Überblicksartikel, der sich mit der Österreichischen Gesellschaft für Arbeitsund Sozialrecht (ÖGARSR) und deren Tagungsthemen bzw. -beiträgen befasst, CERNY (2015).

4 Es soll nicht unerwähnt bleiben, dass man durchaus - unter Bezugnahme auf die Entwicklungen der letzten zwei bis drei Jahrzehnte (Jahrzehnte der >Globalisierungskrise ) - Analogien zu den 1920er und 1930er Jahren herstellen kann. Dies ist in der aka- demischen Landschaft Österreichs aber eher ein Tabuthema.

5 Und wenn es schon für Österreich einen solchen modernen Forschungsstand nicht gibt, dann könnte man sich wenigstens von internationalen Forschungszugängen und -ergebnissen inspirieren lassen. 
sichtigen, vielmehr sollte der Faktor Forschungsinnovation ausschlaggebend sein.

Einen Bericht über die österreichische Sozialrechtsgeschichte und ihre möglichen Forschungsperspektiven zu verfassen, erfordert zuerst einmal die sechte Juristenlandschaft und ihre rechtsgeschichtlichen bzw. sozialrechtsgeschichtlichen Arbeits- und Artikulationsweisen im Schnellverfahren zu präsentieren und sodann selbst die Perspektive des Sozialrechtshistorikers einzunehmen. Dieser Vorgehensweise kommt zugute, dass die Themen, mit denen sich der Autor seit der zweiten Hälfte der 1970er Jahre ${ }^{6}$ befasst hat, immer auch rechtshistorische Aspekte aufwiesen und ihn zur Auseinandersetzung mit rechtsgeschichtlichen Ansätzen angeregt haben. ${ }^{7}$ Innerhalb der Juristenwelt stellen jedenfalls Arbeits- und Sozialrechtshistoriker eine Minderheit dar. ${ }^{\mathbf{8}}$ Mit Blick auf Österreich würde man hier wohl nur den schon verstorbenen Rechtshistoriker Herbert Hofmeister - einen gebürtigen Münchner - benennen. Allerdings zeigt auch seine Publikationsliste, dass die Sozialrechtsgeschichte kein Schwerpunkt seiner Forschung war. Der seinerzeit von ihm verfasste Länderbericht über Österreich entstand im Zusammenhang mit einem umfänglichen Forschungsprojekt, das vom Max-Planck-Institut für ausländisches und internationales Sozialrecht zum Thema »Ein Jahrhundert Sozialversicherung « organisiert worden war. ${ }^{9}$ Folgt man bundesdeutschen Autoren, dann wäre im Bereich des Sozialrechts auch die Kinderund Jugendhilfe (Kinder- und Jugendfürsorge, Jugendwohlfahrt) mit einzubeziehen. ${ }^{\mathbf{1 0}}$
2 Sozialrechtliche Erkenntnislandschaft samt Themenzonen

\subsection{Begriffsgeschichtliches und Sozialrechtsbereiche}

Der längstgediente und heute noch lebende Sozialrechtler Theodor Tomandl hat in seinem Grundlagenbuch zum österreichischen Sozialrecht schon vor langer Zeit das Sozialrecht in den Kontext der »Sozialen Sicherheit" und eines Entwicklungsprozesses gestellt: »Dynamisch gesehen können wir demnach als Sozialrecht jenen Rechtsbereich betrachten, der sich mit der institutionalisierten Hilfe auf Gemeinschaftsbasis zur Deckung sozialer Risiken befasst, sofern diese Hilfe vom Staat eingerichtet oder zumindest gefördert und überwacht wird.«" ${ }^{\mathbf{1 1}}$ Die Trias von Versicherung, Versorgung und Fürsorge ist auch hier aufgeführt, allerdings im Sinne von »Idealtypen, die in reiner Form in der Wirklichkeit kaum vorkommen «. Tomandl erwähnt eine von Zacher in Deutschland ins Spiel gebrachte Unterscheidung nach Vorsorgesystemen, Entschädigungssystemen und Ausgleichsystemen, die er folgendermaßen einschätzte: »Diese Unterscheidung wechselt allerdings stets das maßgebliche Unterscheidungskriterium (Art, Motiv und Ziel der Sicherung), was ihre Handhabung nicht erleichtert, vermag aber in Ergänzung der herkömmlichen Unterscheidung das sozialpolitisch mögliche Spektrum besser zu erhellen. « ${ }^{\mathbf{1 2}}$ In verfassungsrechtlicher Perspektive manifestiert sich in Österreich eine uneinheitliche Kompetenztatbestandslogik, der zufolge das Sozialversicherungsrecht eine Bundessache in Gesetzgebung und Vollziehung war und ist (Art 10 Abs 1
6 Hinzuweisen ist auf das Institut für Wirtschafts- und Sozialgeschichte an der Universität Wien, an dem etliche Mitarbeiter (wie Birgit BologneseLeuchtenmüller, Ernst Bruckmüller, Hannes Stekl, Roman Sandgruber, Peter Feldbauer) schon in der zweiten Hälfte der 1970er Jahre diesbezüglich durchaus sehr aktiv waren und an dem Dissertationsgruppen (z. B. zur Kinder- und Jugendfürsorge) tätig waren, denen auch der Autor angehörte.

7 Hierbei hat sich der Autor in erster Linie allerdings an der bundesdeutschen (und auch französischen) Wissenschaftsmoderne orientiert und sich von dieser inspirieren lassen.
8 Aus den Reihen der Rechtshistoriker hatte etwa Kurt Ebert seine Habilitationsschrift zu einem wahlverwandten Thema des Sozialrechts, nämlich über die Gewerbeordnungsreform (1879-1885) unter Taaffe, veröffentlicht, EBERT (1975).

9 Hofmeister (1981).

10 Dieser Zugang findet sich zum Beispiel in einem der Standardwerke deutschen Sozialrechts, STOLLEIS (2003). Für Österreich wären dann für die Zeit seit dem ausgehenden 19. Jahrhundert von Relevanz: Melinz (1982); Melinz/Ungar (1996); Melinz (2009).

11 Dieses Zitat stammt aus der vierten Auflage, entspricht aber auch der
Formulierung in der Erstauflage aus dem Jahre 1974, TomandL (1989) 3. Der Autor taxiert den Risikobereich mit »Krankheit, der Mutterschaft, der Arbeitslosigkeit, des Alters, der Invalidität, des Arbeitsunfalles, der Berufskrankheit, der Familienlasten und des Todes des Familienerhalters" und der Option von "mischbaren Verfahrensarten«, ebenda., 4. Die jüngste und letzte Auflage (2009) unterscheidet sich im Einleitungskapitel nicht wirklich von älteren Auflagen.

12 Tomandl (1989) 4. 
Z 11 B-VG), während das zweite soziale Netz seit den 1920er Jahren einem Kompetenztatbestand »Armenwesen" und "Mutterschafts-, Säuglingsund Jugendfürsorge« (Art 12 Abs 1 Z 1 B-VG) zugeordnet war und ist. Demzufolge war und ist nur die Bundesgrundsatzgesetzgebung erlaubt, die Ausführungsgesetzgebung und der Gesetzesvollzug sind Angelegenheit der Bundesländer. Der entscheidende Faktor für die spezifische Verlaufsgeschichte des österreichischen Sozialrechts seit Mitte der 1920er Jahre bis heute ist die so genannte »Versteinerungstheorie«. Damit wird eine verfassungsrechtliche Interpretation umschrieben, der zufolge die Rechtsprechung des Jahres 1925 als lähmende Richtschnur für die weitere Sozialrechtsgesetzgebung - insbesondere das Fürsorge- / Sozialhilferecht betreffend - gewirkt hatte.

Im Folgenden wird nach den üblichen Strukturen und Funktionslogiken vorgegangen, d. h. Versicherung (erstes soziales Netz), Versorgung (zentralstaatliches soziales Netz) und zu Beginn gleich das Prinzip der Fürsorge (zweites soziales Netz).

\subsection{Armen(fürsorge)recht - Sozialhilfe}

Obwohl in der vorgeschlagenen Bildsprache das Armen(fürsorge)recht zuerst existierte und somit als erstes soziales Netz bezeichnet werden könnte, wird es hier als zweites soziales Netz definiert, weil es nach der sukzessiven Etablierung von sozialversicherungsrechtlichen Leistungsansprüchen zum Unterstock desselben wurde. Für Österreich fehlt es an ernsthafter Literatur zur Geschichte des zweiten sozialen Netzes (Armenfürsorge-/Fürsorge-/ Sozialhilferecht) aus der Feder von Rechtshistorikern $^{13}$ bzw. universitären Sozialrechtsmitarbeitern. ${ }^{14}$ Von Historikern gibt es für einige Zeitabschnitte den einen oder anderen Beitrag zu diesem
Themenbereich. Hinsichtlich einer Langzeitperspektive der Sozialrechtsentwicklung in Österreich, die zugleich als Interaktionsgeschichte von erstem und zweitem sozialen Netz (seit den 1880er Jahren bis in die Gegenwart) und dabei historischsozialwissenschaftlich, rechtshistorisch sowie politikfeldanalytisch vorgegangen ist und des Weiteren auch Indikatoren aus eigenen Budgetanalysen verwendete, gibt es hierzulande nur einen Vertreter. ${ }^{\mathbf{1 5}}$ Bildsprachlich wie inhaltlich-konzeptionell wird von dem beitragsfinanzierten und mit Rechtsansprüchen versehenen ersten sozialen Netz (Sozialversicherungsschiene) und dem steuerfinanzierten, bedarfsgeprüften und auf Einzelfälle gerichteten zweiten sozialen Netz ausgegangen, wobei beide Netzstrukturen in einer systemfunktionalen Logik interagieren, und zwar bis zum heutigen Tage. Die Entwicklung des Wohlfahrtsstaates lässt sich nicht verstehen ohne Berücksichtigung dieser Interaktionsmuster von Sozialversicherungsstaatlichkeit und der Sozial(arbeits)politik von Bundesländern und Kommunen auf Basis verfassungsrechtlicher Normen und der einschlägigen Judikatur. Bundesländern bzw. den Landtagen kam und kommt im Rahmen des zweiten sozialen Netzes einerseits gesetzgeberische Kompetenz und andererseits sozialverwaltungsrechtliche Handhabung zu. ${ }^{16}$ Die einschlägigen Forschungsergebnisse zeigen sehr deutlich, dass Armut im Kontext kapitalistisch verfasster Gesellschaften nie und nimmer abgeschafft werden sollte, vielmehr funktioniert(e) Armut stets als disziplinierendes und systemintegratives Element im Räderwerk österreichischer Wohlfahrtsstaatlichkeit. ${ }^{17}$

Aus Platzgründen kann keine ausführliche Begründung für das Plädoyer einer Rekonstruktion der Interaktionsgeschichte des ersten und zweiten sozialen Netzes angeboten werden. Aus heutiger
13 Die Habilitationsschrift einer Rechtshistorikerin beschäftigte sich u. a. mit dem Heimatrecht und dem Ausweisungsrecht sowie den einschlägigen Judikaten und weist somit Berührungspunkte mit dem zweiten sozialen Netz auf, ReITER-ZatLOUKAL (2000).

14 Das Kriterium für diese Bewertung ist, ob quellenbasierte Primärforschung vorliegt.

15 Dieses Konzept verfolgt der Autor dieses Beitrags. Die dazu seit 1982 erschienenen Beiträge werden aller- dings fast nie berücksichtigt bzw. zitiert, d. h. das Grundprinzip >guter Forschung, die mit dem Forschungsstand beginnt und sich theoretisch, methodisch oder empirisch positioniert bzw. abgrenzt, ist in Österreich bezüglich des Forschungsbereichs Soziale Politiken nicht gegeben. Aus Platzgründen kann hier keine umfassende Literaturbelegführung angeboten werden. Die Österreichische Historische Bibliographie bietet eine schnelle Online-Option, um alle deutsch- und englischspra- chigen Beiträge des Autors zu identifizieren. Als Überblick brauchbar ist Melinz (2003).

16 Für eine rechtswissenschaftliche Reflexion - obwohl für die Ära der Sozialhilfe-Gesetze seit der ersten Hälfte der 1970er Jahre - brauchbar ist PfeIL (1989) 40 ff. Für den gleichen Zeitraum einer juristischen Zeitgeschichte zum Thema Armut und Verfassung OppITZ (1998).

17 Melinz (2003). 
Sicht macht es einfach Sinn, mit dem Beginn der liberalen Ära (1860 ff.) und somit eines beginnenden Verfassungsstaates und einem Parlamentarismus, der schrittweise eine aufholende Entwicklung durchlebte, zu beginnen, und zwar unter Berücksichtigung des Industrialisierungsprozesses, der von Urbanisierung, Verstädterung und ausgeprägter Mobilität (’der Lohn-Arbeit nach`) geprägt wurde. Das Reichsgemeindegesetz (1862) und vor allem das Heimatgesetz (1863, Novelle 1896) waren vorerst die grundlegenden rechtsnormativen Eckpfeiler, die je nach Kronland (Armen-Landesgesetze etwa) und Kommune hinsichtlich der Armenfürsorge die relevanten Faktoren waren. Erwerbsarbeitslosigkeit war in der Zeit der Habsburgermonarchie (cisleithanische Reichshälfte) ${ }^{\mathbf{1 8}}$ noch kein anerkanntes soziales Risiko und der armenpolizeilichen Logik, d. h. der Vagabundenund Bettlerbekämpfung, zugeordnet, nicht hingegen der kommunalen Armenfürsorge oder irgendeinem öffentlichen Unterstützungsangebot. Das Strafrecht, das Verwaltungsrecht und natürlich auch das Zivilrecht (Unterhaltsleistungen) waren die bestimmenden Rechtsmaterien, das Subsidiaritätsprinzip als Kernelement des Armenrechts prägte das zweite soziale Netz. Die Jahre der demokratischen Ersten Republik (1918-1933/34) brachten zwar eine Bundesverfassung neuen Typs, aber keine entscheidenden Veränderungen für die Rechtsnormen und die Ausgestaltung des zweiten sozialen Netzes mit sich. Die einzige Ausnahme war vorerst die Etablierung eines zentralstaatlichen Arbeitslosenversicherungsrechts im Jahre 1920. In den 1920er Jahren und vor allem nach dem Ausbruch der Weltwirtschaftskrise 1929 lassen sich alle Spielarten rechtsnormativer, sozialverwaltungspraktischer und armenpolizeilicher Umgangsweisen empirisch aufzeigen. Allerdings braucht es in diesem Zusammenhang zwecks angemessener analytischer Interpretation eine gesamtgesellschaftliche Kontextualisierung, wobei der Prozess der Ausschaltung der demokratischen Republik und ihrer Grundlagen zu berücksichtigen wäre. Zudem wird in einer holistischen Forschungsperspektive überdeutlich, wie begrenzt die vielgeliebte und -gelobte Sozialversicherungsstaatlichkeit realiter war und wie sehr die sich entwickelten Identitätsbarrieren und sozialen Grenzen zwischen der traditionellen Armutsbevölkerung und der respektablen Arbeiterklasse in diesen Jahren verflüchtigt haben. Die Politik der Aussteuerung, d. h. das Verschieben von langzeitarbeitslosen Bevölkerungsgruppen aus dem ersten Sicherungsnetz (z. B. Arbeitslosenunterstützung) in die kommunale Fürsorge, bedeutete beispielsweise die Überforderung von sog. (industriellen) Notstandsgemeinden, die ihrem gesetzlichen Auftrag zur Armenfürsorge nicht mehr nachkommen konnten.

Eine besondere Periode auch in diesem Zusammenhang ist die Zeit der Regierungsdiktatur des "christlichen Ständestaates" (1934-1938). Deren Sozialpolitik, nämlich die schlichte Armutsverwaltung mit einem Akzent auf Naturalleistungen statt Geldleistungen und das den verarmten Bevölkerungsteilen aufgezwungene Überlebensmanagement lassen sich mit einem rein rechtshistorischen Untersuchungsdesign nur begrenzt erfassen. ${ }^{19}$ In der darauf folgenden NS-Ära gingen auf Grund der Aufrüstungspolitik und des Krieges die Erwerbsarbeitslosigkeit und die Fürsorgebedürttigkeit objektiv zurück. Zudem wurde das immer als rückständig eingeschätzte Heimatrecht ${ }^{20}$ abgeschafft und das deutsche Fürsorgerecht implementiert, allerdings - entsprechend den NS-Grundsätzen - verknüpft mit repressiven Maßnahmen gegen "Erbkranke« und "Asoziale« und dem Ausschluss von nicht zum »deutschen Volk« zählenden Personengruppen. Im Bereich des Fürsorgerechts lässt sich nach 1945 eine Kontinuität der reichsdeutschen Fürsorgebestimmungen beobachten, mit der charakteristischen Zweiteilung in "allgemeine" und "gehobene" Fürsorge, die bis zu Beginn der 1970er Jahre erhalten blieb. Wie schon in den 1920er Jahren hätte es zu Beginn der Zweiten
18 Bezüglich eines Österreich-UngarnVergleichs bietet sich Melinz/ ZimmermanN (1996) an.

19 Melinz (2003); Melinz (2010a); Melinz (2010b).

$20 \mathrm{Im}$ deutschen Kaiserreich wurde das Unterstützungswohnsitzprinzip letztlich zur Grundlage der Fürsorge. Das Argument, dass deshalb eine effektivere Industrialisierung möglich war, kann nicht als tragfähiges Argument verwendet werden, weil es ansonsten in der österreichischen Reichshälfte der Monarchie vor dem Hintergrund des Heimatrechts keinen wirtschaftlichen Aufschwung hätte geben können. Hierzulande hatten die betroffenen Menschen nur mehr an sozialer Last und Entbehrung schultern müssen. 
Republik wieder die Möglichkeit gegeben, dass der Bund aufgrund einer vorhandenen verfassungsrechtlichen Ermächtigung ein Bundesgrundsatzgesetz zur Fürsorge (»Armenwesen« und »Mutterschafts-, Säuglings- und Jugendfürsorge«) beschließt. Auch diesmal scheiterte das Vorhaben. ${ }^{21}$

Für die Zeit von 1945 bis 1971 liegen umfassende Quellenbestände vor, die für die rechtshistorische Forschung von großem Interesse sein dürften, denn über die sogenannte Verbindungsstelle der österreichischen Bundesländer ist ein Sammelakt überliefert, in dem der Gang der Reformdebatten zur weiteren Ausgestaltung des Sozialhilferechts sichtbar wird, insbesondere die unterschiedlichen Positionen der Bundesländer, des Bundeskanzleramtes sowie des Innen- und des Sozialministeriums. Letztlich kam es auch zu umfassenden Neuregelungen, zwar nicht auf Bundesebene - was aufgrund der verfassungsrechtlichen Kompetenzzuschreibung ja möglich gewesen wäre -, aber doch in neun Landessozialhilfe-Gesetzen in der ersten Hälfte der 1970er Jahre. ${ }^{22}$ Erwähnenswert - weil auch in deutlicher Weise den seinerzeitigen Diskurs dokumentierend - ist eine Tagung von 1973, auf der sich Sozialrechtler und Vertreter der »Praxis« inmitten des Bundesländer-Gesetzgebungsprozesses mit dem Thema »der Fürsorge im Wohlfahrtsstaat « auseinandersetzten. ${ }^{23}$ Die zeitgenössische Wahrnehmung der Landes-Sozialhilfegesetze fiel sehr optimistisch aus; kaum ein Jahrzehnt später, als sich herausstellte, dass Österreich keine Insel der Seligen ist, begann die Ära der Novellierungen des Sozialhilferechts. ${ }^{24}$

\subsection{Sozialversicherungsgesetzlichkeiten}

Wir kommen nun zum dominanten Sozialversicherungsrecht, das für die Sozialrechtslehre bestimmend ist. $\mathrm{Zu}$ Beginn steht die 1981 publizierte Österreich-Studie von Herbert Hofmeister im Vordergrund, weil sie die einzige Langfristuntersuchung zur Sozialversicherungsstaatlichkeit ist, die von einem Rechtshistoriker verfasst wurde. ${ }^{25}$ Die Fallstudie ist selbstverständlich von einer rechtshistorischen Intention geprägt, eine prägnante rechtshistorische Methodik ist aber nicht erkennbar. Es handelt sich letztlich um eine solide verlaufsgeschichtliche Studie, beginnend mit den ersten Arbeiterversicherungs-Stammgesetzen bis hin zum Allgemeinen Sozialversicherungsgesetz (ASVG) und all den Erweiterungen der österreichischen Sozialversicherungsstaatlichkeit der Zweiten Republik bis 1978. Ein besonderer Mehrwert ergibt sich aus den präzisen und klar formulierten Vergleichseinschätzungen in Bezug auf die deutsche Sozialversicherungsentwicklung. Zeitgleich erschien die Habilitationsschrift des Politikwissenschaftlers Emmerich Tálos, der sich ebenfalls mit dem ersten sozialen Netz, der Sozialversicherungsstaatlichkeit, auseinandersetzte. ${ }^{26}$ Das so genannte zweite soziale Netz blieb in beiden Studien ausgeblendet. Solange man innerhalb der Perspektive der Sozialversicherungsentwicklung verbleibt, erscheint dies unproblematisch. Sobald hiermit jedoch gesamtgesellschaftliche und sozialpolitikhistorische Einschätzungen verknüpft werden, geht von einem derartigen Ansatz - so die Position des Autors vor dem Hintergrund eigener Forschungen - die Gefahr aus, dass Bilder und Deutungen erzeugt werden, die nicht wirklich zutreffend sind.

Ganz grundsätzlich ist festzuhalten, dass die NSÄra noch immer den größten Forschungsbedarf aufweist. Das gilt letztlich für beide sozialen Netze. Zum einen haben nicht wenige Menschen auf einer persönlichen Ebene zu Beginn der nationalsozialistischen Herrschaft vom Regimewechsel profitiert, zum anderen wurde - systemimmanent betrachtet - das österreichische Sozialrecht durch die Implementation des deutschen Rechts modernisiert, wobei es sich - dies muss klar dazu gesagt werden - überwiegend um deutsches Recht handelte, welches in der Weimarer Demokratie entstanden war. Um Missverständnissen vorzubeugen:
21 Gescheitert ist ein Bundesgrundsatzgesetz für den Kompetenztatbestand "Armenwesen « (Erwachsenenfürsorge / Sozialhilfe), nicht aber das Bundesgrundsatzgesetz zum zweiten Verfassungskompetenztatbestand, auf dessen Grundlage 1954 das Jugendwohlfahrtsgesetz (BGBI Nr. 99/1954) und weitere neun Länder-Ausführungsgesetze erlassen wurden.

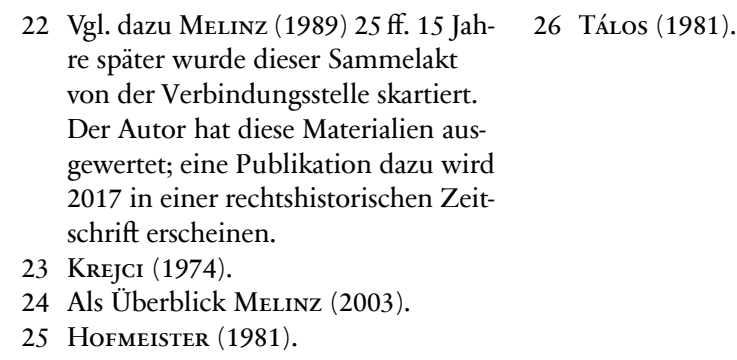

22 Vgl. dazu Melinz (1989) 25 ff. 15 Jah- 26 TÁLos (1981). gewertet; eine Publikation dazu wird 2017 in einer rechtshistorischen Zeitschrift erscheinen.

25 Hofmeister (1981). 
Die rassistisch-menschenverachtenden Radikalisierungspraktiken waren nicht explizit in den Sozialversicherungsgesetzen oder im Fürsorgerecht festgeschrieben. ${ }^{27}$ Die postfaschistische Ära zeichnete sich - nach der jegliche nationalsozialistischen Inhalte aus der Gesetzgebung verschwunden waren - zunächst durch eine Kontinuität des Sozialrechts aus. Im Bereich des ersten sozialen Netzes kam es dann 1955 zu einer österreichischen Lösung, dem bis zum heutigen Tage geltenden Allgemeinen Sozialversicherungsgesetz (ASVG). ${ }^{\mathbf{2 8}}$

Die Geschichte der Sozialversicherung - in all ihren berufsständischen Gruppen- und Gliederungsformen - ist schon lange kein Forschungsthema mehr. Die Jubiläumsliteratur von Sozialversicherungsträgern hatte ohnedies stets ihre wissenschaftlichen Grenzen und stand nie für Forschungsinnovation. Was sonstige historische Befunde betrifft, ist auch hinsichtlich des ersten sozialen Netzes noch einmal auf den Umstand hinzuweisen, dass es streng genommen keine Sozialrechtshistoriker in Österreich gibt, jedenfalls nicht solche, die sich dem ausgehenden 19. und dem 20. Jahrhundert widmen würden. Daher muss auch hier wieder der Blick auf die Historiker gerichtet werden. Als ein Schwerpunkt lässt sich hierbei das Prinzip der Selbstverwaltung in der Sozialversicherung ausmachen. Dieses Thema wurde sowohl als verfassungsrechtliches Thema akzentuiert $^{29}$ als auch unter dem Gesichtspunkt der Arbeitnehmermitbestimmung behandelt. ${ }^{30}$ Arbeiten existieren auch zur Pensionsversicherung [bundesdeutsch: Rentenversicherung]. Als Arbeiterversicherung wurde sie letztlich erst im NS-Staat eingeführt, während sie für Privatbeamte (im modernen Sprachgebrauch: Angestellte) aus politischem
Kalkül bereits 1906 im Vorfeld der Realisierung des Allgemeinen Männer-Wahlrechts zustande gekommen war. Hierzu existiert - nach der lange zurückliegenden, aber gleichwohl umfassenden Dissertation von Andreas Baryli ${ }^{31}$ - eine neuere quellengesättigte Arbeit, die auch die Ausstrahlungen auf die Rechtsentwicklung in Deutschland in den Blick nimmt. ${ }^{32}$

Die Entstehung der Krankenversicherung in Österreich stellt sich dar als eine Transformation von Selbsthilfe-Formen in eine staatlich orchestrierte Versicherung im Jahre 1888. Alexander Prenninger hat diesen Prozess mit guten Gründen im Rahmen einer Fallstudie zu Salzburg untersucht, um die regionalen und lokalen Realitäten besser in den Blick zu bekommen und auch die an Widersprüchen reiche Vielfalt zu identifizieren. ${ }^{33}$

Die Zahl der Nutznießer der ersten Sozialversicherungsgesetze war zunächst sehr gering. Die nachfolgende Einbeziehung von Landarbeitern, Hausgehilfinnen, Gewerbetreibenden und Bauern - im Sinne einer sozialen Sicherheit im »Nachziehverfahren « - wird in einem von Wirtschafts- und Sozialhistorikern herausgegebenen Sammelband behandelt; ${ }^{34}$ eine jüngere Arbeit konzentriert sich auf die Entwicklung der bäuerlichen Sozialversicherung im 20. Jahrhundert. ${ }^{35}$

Die Arbeitslosenversicherung nimmt eine gewisse Sonderstellung ein, welche sie auch schon seit ihrer Einführung in den 1920er Jahren hat und die in ihrer besonderen Konstruktion begründet ist. Denn die Arbeitslosenversicherung ist in Form eines 2-Phasen-Modells organisiert: Einer beitragsfinanzierten Sozialversicherungsleistung in der ersten Phase (Arbeitslosenunterstützung/Arbeitslosengeld) folgt eine zweite Phase, die nicht mehr
27 Im Zusammenhang mit dieser Modernisierungsthese muss auch auf das Jugendwohlfahrtsrecht hingewiesen werden.

28 Hofmeister (1981); TÁlos (1981). In den letzten Jahren wurden vermehrt Studien im Auftrag des Hauptverbandes der österreichischen Sozialversicherungsträger veröffentlicht, wobei zumeist eine Persönlichkeit im Zentrum steht und die dazugehörige Zeitspanne sozialversicherungsgeschichtlich bearbeitet wurde. Die Zeiträume beziehen sich auf die Erste und die Zweite Republik. Teilweise finden sich hier sehr respektable Quellen und manchmal rechtsge- schichtlich relevante Informationen, insgesamt ist diese Schriftenreihe aber eher von einem historisch-politikwissenschaftlichen Zugang geprägt.

29 Zum Beispiel ÖHLINGer (2002). Auf die konfliktbeladene »Lex Salmutter" bzw. die Ablösung des (sozialdemokratischen) Hauptverbandspräsidenten Hans Salmutter 2001 durch die ÖVP-FPÖ-Regierungskoalition kann hier nicht eingegangen werden. Hier drängen sich historische Analogien zu den 1930er Jahren auf.

30 Von den Anfängen bis 1918 grundlegend GrandL (2004).

31 BARYLI (1977).
32 Кім (2010)

33 Prenningers Diplomarbeit stammt aus dem Jahre 2001, eine prägnante Zusammenfassung bietet Prenninger (2005).

34 BRucKMüLler/SANDGRUber/STEKL (1978).

35 Siegl/Steiner (2010). 
dem reinen Äquivalenzprinzip einer Sozialversicherungsleistung entspricht (Notstandsaushilfen / Notstandshilfe); in rechtshistorischer Perspektive besteht hier noch etlicher Forschungsbedarf.

Im Hinblick auf historisch-empirische und holistisch-wohlfahrtsstaatliche Forschungsperspektiven ist hingegen auf einige Publikationen zu verweisen, die anhand des Arbeitslosenversicherungsrechts zeigen, wie sensibel in diesem Bereich auf die Realitäten der Wirtschaft und des Arbeitsmarkts reagiert wurde. ${ }^{36}$ Seit der Etablierung einer Pflichtversicherung im Jahre 1920 hat dieser Umstand die häufigen Novellierungen des Arbeitslosenversicherungsrechts am meisten geprägt. ${ }^{37}$

Die Forschung konzentriert sich im Regelfall auf die lohnarbeitszentrierte Sozial(versicherungs)staatlichkeit. Es gibt aber auch Ausnahmen, wie die jüngst abgeschlossene Dissertation eines promovierten Juristen und nunmehr promovierten Historikers zeigt, der sich mit dem Forschungsdesiderat der Entstehung der Sozialversicherung des österreichischen Notariats auseinandergesetzt hat. ${ }^{38}$ Wolfgang Gabler bietet mit seiner umfassend quellenbasierten Forschungsleistung ein buntscheckiges Bild einer langwierigen und wechselhaften Verlaufsgeschichte von einer freiwilligen Selbstversicherung hin zu einer gesetzlichen Pflichtversicherung (1926). In einer sozialrechtshistorischen Perspektive, die auch gegenwartsbezogene Erkenntnisinteressen einschließt, lassen sich drei Aspekte dieser Arbeit hervorheben, die auch von allgemeiner Bedeutung sind. Erstens handelt es sich bei den Notaren um Angehörige der Gruppe der >freien Berufe $<$ und in Form der Notariatskandidaten um vorerst einmal Privatangestellte. Zweitens wird historisch-empirisch am Beispiel der genannten zweiphasigen Berufsgruppe offenkundig, dass die privaten Lösungen gesellschaftlicher und sozialer Sicherung nicht nachhaltig gelingen. ${ }^{39}$ Aus heutiger Sicht interessant ist zudem der Tatbestand, dass eine Berufsgruppe aus den Reihen des Bürgertums schon aus Eigeninteresse dem Umlageprinzip den Vorzug vor einer
Kapitaldeckungsstrategie im Bereich der eigenen Sozialversicherung gegeben hatte. Drittens zeigt sich in dieser Studie - und in jeder anderen Studie mit Langzeitperspektive -, dass ein (sozial-) rechtsgeschichtlicher Forschungsansatz die gesellschaftsgeschichtlichen Rahmenbedingungen und die Zeit-Raum-gebundenen Akteurskonstellationen berücksichtigen muss, um die rechtsnormativen und sozial- und armutspolitischen Konsequenzen hinreichend präzise erfassen und einordnen zu können.

\subsection{Zentralstaatliche Versorgungsgesetzgebung}

Neben dem ersten und zweiten sozialen Netz etablierten sich im Zuge des Ersten Weltkriegs diverse allgemein steuerfinanzierte Versorgungsbereiche, die in zentralstaatlicher bzw. bundesstaatlicher Verantwortung liegen. Die sozialrechtlichen Leistungsangebote hatten nichts mit einer armenfürsorgerischen Bedarfsprüfung zu tun, sie waren vielmehr mit einem Rechtsanspruch bei Vorliegen bestimmter Voraussetzungen (»Schädigungen«) verknüpft.

>Vater Staat< als Kriegsherr hatte vor allem im Kontext des Ersten Weltkriegs Versorgungsleistungen für Kriegsinvalide, Kriegerwitwen und Kriegswaisen eingeführt, ergänzt um staatliche Unterhaltsleistungen für Familien von Soldaten. In den zwei Weltkriegen haben sich eigenständige Versorgungskomplexe herausgebildet, zu denen in jüngster Vergangenheit Forschungsarbeiten mit auch rechtshistorischer Relevanz erschienen sind. Zum Ersten Weltkrieg und seinen Folgen haben Verena Pawlowski und Harald Wendelin ein umfangsreiches Werk ${ }^{40}$ publiziert, das sowohl die Vorgeschichte des Militärversorgungsgesetzes (1875) als auch die Etablierung des Invaliditätsentschädigungsgesetzes des Jahres 1919 und die in weiterer Folge entstandenen Gesetzesnovellierungen und ihre Implikationen aufgearbeitet hat. Für sozialrechtshistorische Forschungsinteressen ist insbesondere die Herausarbeitung der begriff-
36 Melinz (2008); Melinz (2010b).

37 Dies gilt nicht nur für die Zwischenkriegszeit, sondern auch für die Zeit nach 1945, MelinZ (2003); MeLinZ (2008).

38 Gabler (2015).

39 Es geht hier nicht darum, dass sie nicht gelungen sind, sondern sol- cherart Konstruktionen können einfach auf Grund von Konstruktionsfehlern nicht nachhaltig gelingen. Das ist nicht das Argument von Gabler, vielmehr ist es eine wirtschaftshistorische Erfahrungstatsache. 40 Pawlowski/Wendelin (2015). 
lichen Spielarten der Kriegsbeschädigtenfürsorge oder der Kriegsopferfürsorge, einschließlich der nicht-soldatischen Opfer (z.B. Kriegerwitwen, Kriegswaisen), interessant. Allerdings ist auch vor unzutreffenden Deutungen zu warnen, wenn beispielsweise die erwähnte Studie mit der Metapher »Kriegsopferfürsorge als Beginn moderner wohlfahrtsstaatlicher Politik « operiert. ${ }^{41}$ Einerseits kann man solcherart Interpretation nur wagen, wenn man den Forschungsstand zur Geschichte der Sozialen Politiken ignoriert. Die »Kriegsopferfürsorge« ist eben nur das dritte Feld bzw. das soziale Netz für bestimmte Zielgruppen. Andererseits ist der Erste Weltkrieg in der Tat jene Periode, in der es auch bei sozialpolitisch relevanten Bereichen zu rechtsnormativen wie rechtspolitischen Umwälzungen kam, kurzum: die Möglichkeit bestimmte liberal-bürgerliche Rechtskulturen zu revolutionieren. Dies betrifft beispielsweise etwa den Kinderschutz und die Jugendfürsorge, die in politischer wie institutioneller Hinsicht neue Wege einschlugen.

Der Zweite Weltkrieg bzw. das NS-Regime führten nicht zum Verschwinden der zentralstaatlich verantworteten klassischen Kriegsopferversorgung nach 1945. Neben dieser Kontinuität lässt sich aber auch die Herausbildung eines neuen und zusätzlichen Rechtskomplexes - in Gestalt des Opferfürsorgegesetzes (OFG) von 1947 - beobachten. Ursprünglich ging es nur um die »Fürsorge für die Opfer des Kampfes um ein freies, demokratisches Österreich «. In einer ersten Version der Erweiterung wurden die »Opfer politischer Verfolgung « einbezogen; in beiden Fällen konnten auch bestimmte Hinterbliebene Leistungen beanspruchen. Im Rahmen der Tätigkeit der »Österreichischen Historikerkommission«, die sich mit dem Vermögensentzug während der NS-Zeit sowie Rückstellungen und Entschädigungen seit 1945 in Österreich beschäftigte und entsprechende Forschungsprojekte beauftragt hatte, sind auch drei hervorragende sozialrechtshistorisch relevante
Publikationen entstanden. ${ }^{\mathbf{4 2}}$ Eine Studie, verfasst von einem gegenwartsbezogenen Sozialrechtler, der wirklich alle methodischen und analytischen Facetten ausgeschöpt hat, sticht dabei hervor. Sie befasst sich mit der Entschädigung von Opfern des Nationalsozialismus im österreichischen Sozialrecht und schließt auch die zahlreichen Novellierungen bis zum Jahrtausendbeginn ein. Wie schon in der Ersten Republik verschwindet auch in der Zweiten Republik im Bereich der sozialrechtlichen Entschädigung die Materienverquickung von Opferfürsorge und Begünstigung im Pensionsrecht nicht. ${ }^{43}$ Aus Platzgründen soll es hier genügen, einige Stichworte zentralstaatlicher Versorgungspolitik zu nennen: Kriegsopferversorgungsgesetz 1957, Heeresversorgungsgesetz, Kriegsgefangenenentschädigungsgesetz, Rentenleistungen für Contergan-Geschädigte und Verbrechensopfergesetz, Impfschadengesetz. Das Bundespflegegeldgesetz im Unterschied zu Deutschland kein Versicherungskonstrukt, sondern eine universelle staatliche Unterstützungsleistung - wäre in diesem Zusammenhang ebenfalls noch aufzuführen.

\section{Forschungsperspektiven}

In Österreich hat zumindest bis 1945 der Blick über die Grenzen nach Deutschland eine große Rolle gespielt. In diesem Zusammenhang könnte man sich analog zum bundesdeutschen Vorbild ein Projekt "Quellensammlung zur Geschichte der Sozialpolitik « für die Zeit der Habsburgermonarchie (1860-1918) ${ }^{\mathbf{4 4}}$ und darüber hinaus vorstellen, um hierzulande aufzuholen. Eine weitere sozialrechtsgeschichtliche Forschungsoption wäre eine vertiefende Bearbeitung der gemeinsamen Jahre der NS-Ära, wobei es innerhalb des "völkischen" Wohlfahrtsstaates (Sachße/Tennstedt) zum einen um die Modernisierungs- und Verbesserungseffekte im Bereich der Sozialversicherung wie des Fürsorgerechts gehen könnte, zum anderen um die
41 Pawlowski/Wendelin (2015) 14.

42 Vgl. zur Systematik und Rechtsanwendung PfeIL (2004); zur Vollzugspraxis des Opferfürsorgegesetzes BERGER et al. (2004) sowie hinsichtlich der praktischen Vollziehung des einschlägigen Sozialrechts im Bereich des Allgemeinen Sozialversicherungsgesetzes Dimmel et al. (2004).
$43 \mathrm{Zu}$ diesem Themenkomplex ist noch die sozialrechtshistorische Arbeit von GrUber (1998) zu erwähnen.

44 Eine interaktionsgeschichtliche Perspektive für Ungarn mit Vergleichsperspektive zu Österreich. ZimmermanN (2010). 
Frage nach Kontinuität und Bruch im Hinblick auf die Nachkriegsepoche. Weiterhin sollte man sich mit der rechtsgeschichtlich bislang vernachlässigten Judikatur der höchstgerichtlichen Instanzen beschäftigen, und zwar alle sozialrechtlichen Themenbereiche betreffend. ${ }^{45}$

Da Österreich 1995 EU-Mitgliedsstaat wurde, wären auch in diesem Zusammenhang entsprechende sozialrechtsgeschichtliche Forschungsperspektiven anzudenken. Obgleich der folgende $\mathrm{Zu}$ gang möglicherweise nicht mehrheitsfähig ist, so ist dennoch evident, dass die armutsgetriebene Mobilität von EU-Bürgern aus den neuen EU-Mitgliedsstaaten und die damit verbundenen sozialrechtlichen wie auch sozialpolitischen Herausforderungen ein zeitgeschichtliches, rechtsgeschichtliches wie politikwissenschaftliches Forschungsinteresse wecken sollten. Ähnliches gilt für die von der aktuellen Hektik der Tagespolitik getriebenen sozialrechtlichen bzw. rechtspolitischen Entscheidungen zum Flüchtlingsthema und für die Frage der restriktiven Mindestsicherungspolitik (Sozialhilfe bzw. neuerdings Bedarfsorientierte Mindestsicherung) in zahlreichen Bundesländern. ${ }^{\mathbf{4 6}}$ Höchstgerichtliche Judikatur in Österreich und des Europäischen Gerichtshofs wird nicht lange auf sich warten lassen. Jedenfalls sollte bzw. darf Sozialrechtsgeschichtsschreibung sich nicht auf Themen beschränken, die mindestens ein halbes Jahrhundert zurückliegen. Und zu guter Letzt stellt sich natürlich auch die Frage nach der disziplinären Wissenschaftsverortung: reine Rechtsgeschichte, interdisziplinäre, intradisziplinäre oder transdisziplinäre Forschungskonzepte und -strategien? Quo vadis österreichische (Sozial-)Rechtsgeschichte?

\section{Bibliographie}

- Berger, Karin et al. (2004), Vollzugspraxis des »Opferfürsorgegesetzes«. Analyse der praktischen Vollziehung des einschlägigen Sozialrechts, Wien, München

- Bruckmüller, Ernst, Roman Sandgruber, Hannes Stekl (1978), Soziale Sicherheit im Nachziehverfahren. Die Einbeziehung der Bauern, Landarbeiter, Gewerbetreibenden und Hausgehilfen in das System der österreichischen Sozialversicherung, Salzburg

- Cerny, Josef (2015), Der Einfluss der »Zeller Tagung« auf die Entwicklung des Arbeitsrechts und des Sozialrechts, in: DRdA 5a, 379-395

- Dimmel, Nikolaus et al. (2004), Analyse der praktischen Vollziehung des einschlägigen Sozialrechts hinsichtlich der Vollzugspraxis im Bereich der $\$ \$ S 500 \mathrm{ff}$ ASVG, Wien, München

- Ebert, Kurt (1975), Die Anfänge der modernen Sozialpolitik in Österreich. Die Taaffesche Sozialgesetzgebung für die Arbeiter im Rahmen der Gewerbeordnungsreform (1879-1885), Wien

- Gabler, Wolfgang (2015), Die Entstehung der Sozialversicherung des österreichischen Notariates. Ein Berufsstand auf dem Weg von der freiwilligen Selbstversicherung zur gesetzlichen Pflichtversicherung, Dissertation Universität Wien, Wien

- Grande, Robert (2004), Die Geschichte der Selbstverwaltung und die Arbeitnehmermitbestimmung in der österreichischen Sozialversicherung. Teil 1: Von den Anfängen bis 1918, Wien

- Gruber, Bernhard W. (1998), Hinterbliebenenleistungen in der österreichischen Sozialversicherung von 1888 bis 1955 , Wien

- Krejci, Heinz (1974), Probleme der Fürsorge und Sozialhilfe im Wohlfahrtsstaat mit den österreichischen Sozialhilfegesetzen im Anhang, Wien

- Hofmeister, Herbert (1981), Landesbericht Österreich, in: Köhler, Peter A., Hans F. Zacher (Hg.), Ein Jahrhundert Sozialversicherung in der Bundesrepublik Deutschland, Frankreich, Großbritannien, Österreich und der Schweiz, Berlin, 445-730

- Kim, Byung Ho (2010), Die Entstehung der Pensionsversicherung für die Angestellten in Österreich mit ihrem Einfluss auf Deutschland und ihre historische Bedeutung, Dissertation Universität Wien, Wien

45 Für einige Sozialversicherungsbereiche finden sich in der gegenwartsbezogenen Sozialrechtsliteratur solcherart Judikatur-Auswertungen. Für eine geschichtliche Auswertung bedürfte es auf spezielle Topoi und längere Untersuchungszeiträume ausgerichteter Forschungsbeiträge.
46 All die quellenbasierten Forschungserfahrungen des Autors in den letzten Jahrzehnten zum Themenkreis der Sozialen Politiken zeigen, dass es immer wieder um Déjà-vu-Erlebnisse geht. 
- Melinz, Gerhard (1982), Hilfe, Schutz und Kontrolle. Versuch zur historischen Genese der öffentlichen »Jugendfürsorge« in Österreich, unter besonderer Berücksichtigung von Wien (1880-1914), Dissertation Universität Wien

- Melinz, Gerhard, Gerhard Ungar (1996), Wohlfahrt und Krise. Das Beispiel Wiens 1929-1938, Wien

- Melinz, Gerhard, Susan Zimmermann (1996), Armenfürsorge, Kinderschutz und Sozialreform in Budapest und Wien 1870-1914, in: Geschichte und Gesellschaft, H. 3, 338-367

- Melinz, Gerhard (2003), Von der Armenfürsorge zur Sozialhilfe: Zur Interaktionsgeschichte von »erstem« und »zweitem« sozialem Netz in Österreich am Beispiel der Erwachsenenfürsorge im 19. und 20. Jahrhundert, Habilitationsschrift Universität Wien, Wien

- Melinz, Gerhard (2008), Jenseits des Reichtums. Existenzbedingungen zwischen Lohnarbeit, Arbeitslosigkeit und Armut in Niederösterreich 1918-1995, in: Melichar, Peter, Ernst Langthaler, Stefan Eminger (Hg.), Wirtschaft. Niederösterreich im 20. Jahrhundert. Bd. 2, Wien, Köln, Weimar, 469-506

- Melinz, Gerhard (2009), In the Interest of Children: Modes of Intervention in Family Privacy in Austria (1914-1945), in: Hauss, Gisela, Dagmar Schulte (Hg.), Amid Social Contradictions. Towards a History of Social Work in Europe, Opladen, Farmington Hills, 203-224

- Melinz, Gerhard (2010a), Erwerbsarbeitslosigkeit und Armut im Spannungsfeld sozial- und armutspolitischer Strategien in Österreich (1920-1938), in: Bruckmüller, Ernst (Hg.), Armut und Reichtum in der Geschichte Österreichs, Wien, München, $141-165$

- Melinz, Gerhard (2010b), Zwischen Opferstatus und »Überlebensmanagement«. Arbeitslose in der Weltwirtschaftskrise (1929-1933), in: ÖGL, Heft 1, 52-63

- Öhlinger, Theo (2002), Die Bedeutung der Selbstverwaltung in der Sozialversicherung, in: DRdA, Heft 3, 191-210

- Oppitz, Florian (1998), Armut und Verfassung. Die Rechtslage in Österreich, in: Hofmann, Rainer et al. (Hg.), Armut und Verfassung. Sozialstaatlichkeit im europäischen Vergleich, Wien, 161-180

- Pawlowsky, Verena, Harald Wendelin (2015), Die Wunden des Staates. Kriegsopfer und Sozialstaat in Österreich 1914-1938, Wien, Köln, Weimar

- Pfeil, Walter (1989), Österreichisches Sozialhilferecht. Systematische Kommentierung der Landes-Sozialhilfegesetze, Wien

- Pfeil, Walter (2004), Die Entschädigung von Opfern des Nationalsozialismus im österreichischen Sozialrecht, Wien, München

- Prenninger, Alexander (2005), Probleme der sozialen Krankenversicherung in der take-off-Periode (1888-1919), in: Ders. (Hg.), Mercy or Right. Development of Social Security Systems, Leipzig, 45-79

- Reiter-Zatloukal, Ilse (2000), Ausgewiesen, abgeschoben: eine Geschichte vom ausgehenden 18. bis ins 20. Jahrhundert, Frankfurt/M.

- Reiter-Zatloukal, Ilse, Christiane Rothländer, Pia Schölnberger (Hg.) (2012), Österreich 1933-1938. Interdisziplinäre Annäherungen an das Dollfuß-/Schussnigg-Regime, Wien, Köln, Weimar

- Siegl, Gerhard, Guenther Steiner (2010), Ja, jetzt geht es mir gut ... Entwicklung der bäuerlichen Sozialversicherung in Österreich, Wien

- Stolleis, Michael (2003), Geschichte des Sozialrechts in Deutschland. Ein Grundriß, Stuttgart

- TÁLos, Emmerich (1981), Staatliche Sozialpolitik in Österreich. Rekonstruktion und Analyse, Wien

- Tomande, Theodor (1989), Grundriß des österreichischen Sozialrechts, 4., vollständig neu bearbeitete Auflage, Wien

- Zimmermann, Susan (2010), Armen- und Sozialpolitik in Ungarn im Vergleich zu Österreich, in: Harmat, Ulrike (Red.), Die Habsburgermonarchie 1848-1918. Bd. IX: Soziale Strukturen, Teilband 1/2: Von der Stände- zur Klassengesellschaft, Wien, 1465-1535 